\title{
HIGH ENERGY SYNCHROTRON X-RAY MEASUREMENTS OF 2D RESIDUAL STRESS STATES IN METAL MATRIX COMPOSITES
}

\author{
Alexander M. Korsunsky, Karen E. Wells \\ Department of Mechanical, Materials and Manufacturing Engineering, \\ University of Newcastle, Newcastle upon Tyne NE1 7RU, United Kingdom
}

Keywords: X-ray diffraction, synchrotron, residual stress measurement, metal matrix composites

\begin{abstract}
The advent of the high intensity, high energy synchrotron X-ray beamlines at the European Synchrotron Radiation Facility at Grenoble has presented the residual stress measurement community with some unprecedented opportunities, as well as challenges. In order to explore the capabilities and limitations of the new instruments, we have studied residual and in situ loading strain distributions in a number of metal matrix composite systems, using BM16, the Powder Diffraction beamline, and ID11, the Materials Science beamline, at ESRF. In this paper, we discuss the methods of data analysis, error estimation, and measurement interpretation, and present some results for both particulate and fibre-reinforced materials.
\end{abstract}

\section{INTRODUCTION}

Diffraction strain measurement is a unique experimental tool available to the stress analyst, since it is capable of providing highly accurate direct data about the state of elastic deformation, both for single crystals and polycrystalline materials. The basis of strain measurement is Bragg's law of diffraction, which relates the angle between the incident and the diffracted beams, $\theta$, to the radiation wavelength, $\lambda$, and the lattice spacing, $\mathrm{d}$, by $\lambda=2 d \sin \theta$.

Assuming that the incident beam energy (wavelength) and direction are suitably well defined, this relationship provides a recipe for measuring the lattice spacing by determining precisely the direction of the diffracted beam. In the hypothesis of small elastic deformations of the lattice, which applies almost uniformly to the metallic and ceramic materials, the strain with respect to some reference state $d_{0}$ (which does not have to be assumed strain-free) is given by

$$
\varepsilon=\Delta d / d=-\cot \theta \Delta \theta \text {, }
$$

where $\Delta d=d-d_{0}$. In view of the fact that angular positions can often be measured to very high precision, this suggests that very high strain resolutions should be achievable by X-ray diffraction. In practice, however, it is possible to identify a list of factors that act to delimit the measurement accuracy, and can be overcome to a greater or lesser extent depending on the experimental set up.

The choice of radiation energies in conventional laboratory X-ray is limited to that characteristic of electron transitions between core levels in atoms of the target. Hence, measuring strain in a class of materials with certain lattice spacings requires suitable sources to be used. The collimation geometry used in laboratory X-ray equipment is often such that significant beam divergence is present, causing peak broadening. This can be partially overcome using focusing arrangements, such as in the cylindrical Guinier cameras; however, these instruments are often unsuitable to stress measurements. In most cases maximisation of flux requires that radiation from a source be directly incident on the sample. A somewhat polychromatic beam diffracting on an ideal sample would produce a peak of finite width, thus limiting the instrumental resolution. Another important limiting factor in the application of laboratory X-ray to stress measurement is the combination of the relatively low intensity of the incident beam with the high absorption coefficients exhibited by most engineering materials. Consequently, if reasonable counting times are to be achieved, the surface 'spot size' for reflection mode measurements can not be reduced below several 
$\mathrm{mm}^{2}$, while measurements in transmission mode are largely unrealistic except for very thin samples (up to several tens of $\mu \mathrm{m}$ ).

Instrumental arrangements available at synchrotron sources such as ESRF are capable of providing significant improvements in terms of beam intensity and energy, monochromatism, parallelism, as well as the spatial and temporal resolution. Of course, depending on the aims of any particular experiment, the instrumental arrangements can be optimised for best accuracy in the suitable parameters. In the present paper we discuss strain measurements made in metal matrix composites using two different instruments at the ESRF: BM16, the powder diffraction beamline, and ID11, the materials science beamline. In each case, we describe the instrument configuration, data collection and error analysis algorithms and implementation, and demonstrate some of the results obtained. We conclude by comparing the performance and discussing the possibilities for the future.

\section{EXPERIMENTAL}

The experimental configuration used at the BM16 powder diffraction beamline is similar to that of a two-circle diffractometer often used in conventional laboratory X-ray measurements. The incident beam is guided through a horizontal evacuated pipe tangentially outwards from the synchrotron ring. The photon energy can be selected in the range $5-40 \mathrm{keV}$ (wavelengths of 2.5 to $0.31 \AA$ ) using a Ge single crystal monochromator.

Using a pair of stepper motors, light sensitive paper and a theodolite, the diffractometer axis can be aligned with the incident beam, which for this purpose can be collimated to a vertical aperture $0.1 \mathrm{~mm}$. The use of up to 6 pairs of horizontal and vertical collimating slits separated by distances of several meters ensures very low divergence of the beam incident on the sample.

In our experiments, we have used two specimen mounting arrangements. In the first, the specimen is mounted on an XY translation stage, which in turn is attached to the smaller circle. In the second, the specimen was mounted on a separate table, which provided XZ translation, but no $\theta$ rotation. In both cases additional alignment was required in order to locate the incident beam 'spot' on the sample.

The bigger circle on the BM16 diffractometer houses a bank of 9 detectors separated by angular distances of about $2^{\circ}$. Immediately in front of the detectors is a bank of 9 analyser crystals, which must be aligned so that the detectors are in Bragg position with respect to the beam off the sample. The analyser arrangement insures very low angular acceptance of the detector bank, so that in practice the diffracted beam can also be thought of as parallel.

The data collection is performed by continuous scanning of the detector bank, accompanied by data output from each detector at given rate. Precise position of the bigger circle is also stored by recording the signal from stepper motor encoders. Scans in excess of two degrees produce overlapping traces from different detectors. The way in which the data are combined is described in the following section.

The combination of collimation and analyser crystal arrangement defines a sampling volume within the specimen as a parallelepiped. Its vertical cross-section is very eccentric at low diffraction angles, but tends to a rectangle for $2 \theta=90^{\circ}$. Spatial scanning is performed by moving the specimen with respect to the beam, which in principle allows the strain variation in three dimensions to be mapped. The practical limitations arising due to absorption and sampling volume shape are discussed in the following sections. As an alternative to the scanning arrangement described above, we have also used a two-dimensional image plate phosphor detector, which can be read and digitised using a special device, producing an output similar to that of a CCD camera.

The optics characteristics of the ID11 materials science beamline allow photon energies up to $90 \mathrm{keV}$ (wavelengths down to $0.14 \AA$ ) to be used. The high penetrability of the radiation allows strain measurements to be made on thick specimens (e.g. up to $20 \mathrm{~mm}$ aluminium plate) in transmission mode. The data collection strategy we employed on this instrument was similar to that conventionally used to obtain Debye-Scherrer powder diffraction ring patterns. After undergoing monochromation and collimation in the way similar to that described above for BM16, the incident beam is located on the specimen, which is mounted on a translation table XY (i.e. in the plane normal to the beam). A lead beam stop is placed in the way of undiffracted beam in order to protect the two-dimensional detector. The detector can be placed co-axially with the beam, and translated along the beam ( $\mathrm{Z}$ axis) to a position which allows the desired number of diffraction rings to be included in the pattern. 

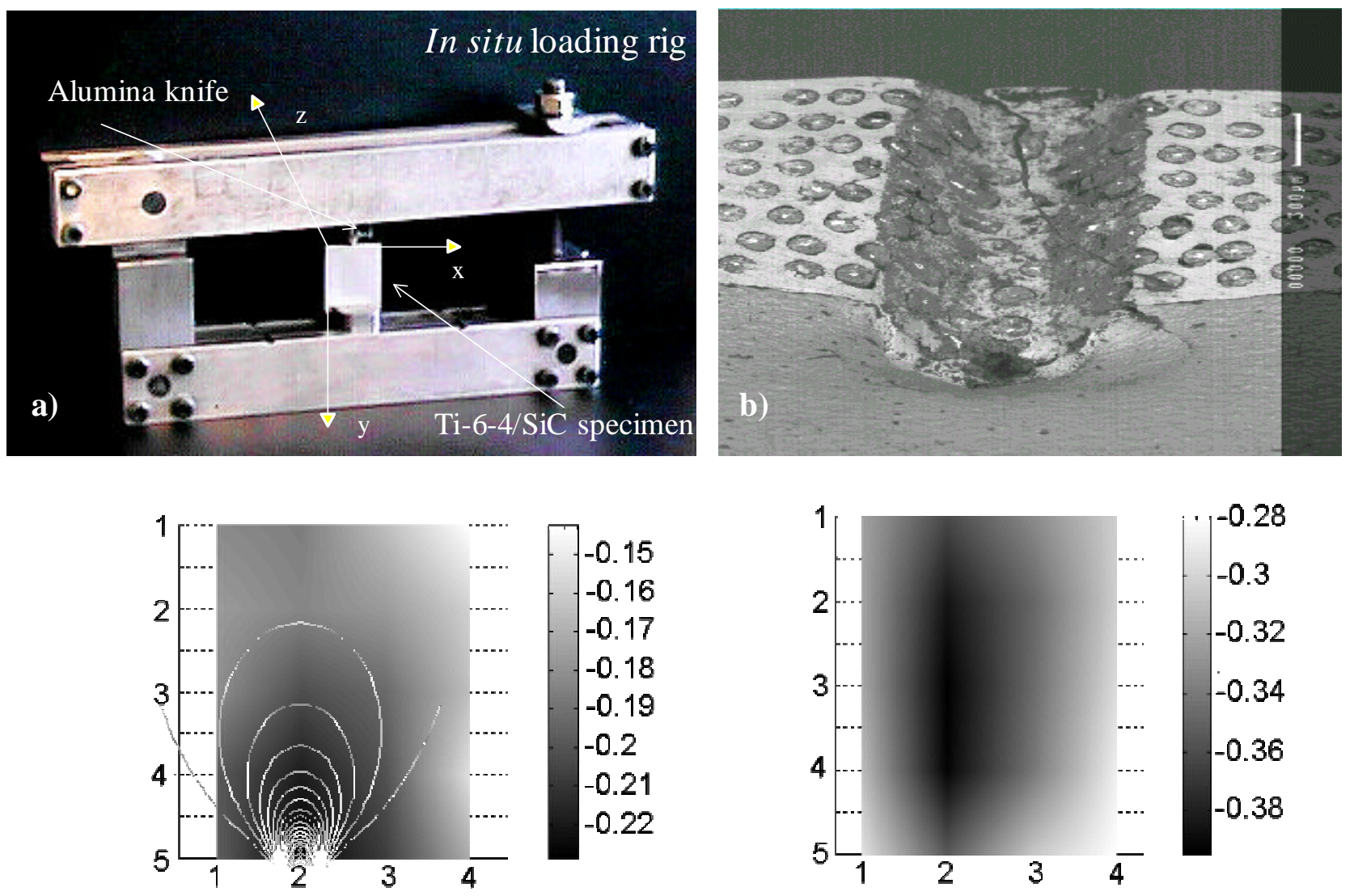

(c)

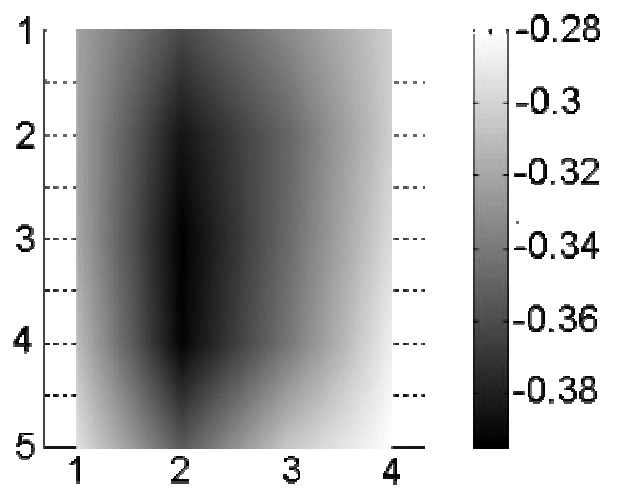

(d)

Fig.1. Analysis of contact loading in Ti-6-4/SiC fibre reinforced composite on BM16: a) the loading arrangement, b) SEM micrograph of an indent, and the measured strain in c) the matrix and d) the fibres. Dotted lines show the strain given by the Hertzian model in a homogeneous material.

The detector we used on ID11 in the present investigation was a Princeton CCD camera having the resolution of $1152 \times 1242$ pixels, pixel size of approximately $30 \mu \mathrm{m}$, and pixel intensity range of 0 to 64,000 counts. In our experiments the time needed to collect the diffraction data was comparable to the time required by the control circuitry to read off and store the camera output (several seconds).

It is clear that, in principle, two-dimensional patterns contain large amounts of detailed information about the state of strain within the sampling volume. However, alongside the clear advantages of data collection using a CCD camera, such as the fast digital output, it is important to be aware of its shortcomings. An example is the lack of confocality between the camera surface and the diffracted beam, which leads to image distortion. Special calibration techniques have been developed at ESRF [1] in order to eliminate this source of errors.

The methodology for data interpretation and error estimation and the algorithms used in our experiments to extract strain information are addressed in the following section.

\section{DATA AND ERROR ANALYSIS}

Let us assume that a sampling volume has been defined within the specimen by the intersection of the incident and diffracted beams. This sampling volume should be small enough to achieve the desired spatial resolution, but also sufficiently large to include a significant number of suitably orientated grains. 

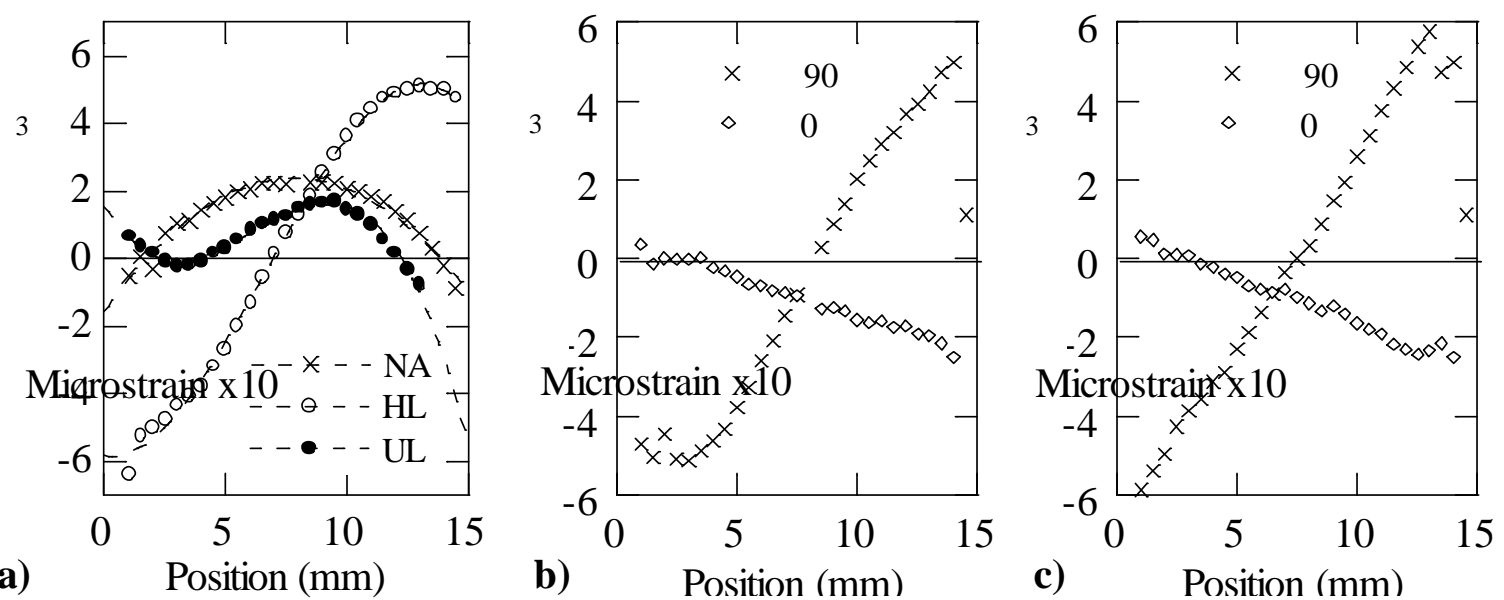

Fig.2. Strain variation during plastic bending of $\mathrm{Al} / \mathrm{SiC}$ specimen: a) profiles of the strain component $\varepsilon_{\mathrm{yy}}\left(90^{\circ}\right.$ with $\mathrm{x}$-axis) measured in the reference condition (NA), under maximum applied bending moment (HL), and upon unloading (UL); b) increment of strain $\varepsilon_{\mathrm{yy}}$ along the bar $\left(90^{\circ}\right)$ and $\varepsilon_{\mathrm{xx}}$ across the bar $\left(0^{\circ}\right)$ during loading (HL-NA); c) increment of strain $\varepsilon_{\mathrm{yy}}$ along the bar $\left(90^{\circ}\right)$ and $\varepsilon_{\mathrm{xx}}$ across the bar $\left(0^{\circ}\right)$ during unloading (HL-UL).

Two-dimensional strain scanning in engineering components can be understood in one of the following two ways:

1. 2D strain state 'at a point' can be described, for example, in terms of the orientation of the principal axes and the magnitude of principle strains (2D-strain measurement).

2. A single component of direct strain can be measured at a sequence of points which form a $2 \mathrm{D}$ mesh within the component (strain 2D-mapping).

In order to avoid confusion, we use a dash to indicate clearly whether the $2 \mathrm{D}$ property is associated with each pointwise strain measurement, or with the resulting map. Of course, it is also possible to create a 2D-map of 2D-strain; however, in the present study we only report (a) 2Dmapping of a single strain component, carried out on BM16, and (b) line scanning of 2D-strain on ID11.

An important issue in strain measurement is the correct estimation of errors associated with each detector counting event, and their propagation throughout the analysis procedure, allowing the accuracy of the strain data to be evaluated. In the most general case on BM16 output must be combined from nine detector channels possessing different sensitivity. The incident beam intensity is known to fluctuate significantly over the scan time, and must therefore be continuously recorded using a monitor counter. A suite of data and error analysis programmes taking all these considerations into account was written by Gavin Vaughan [2]. The software produces an equivalent single line scan, and allows it to be fit for extract peak positions, intensities, widths, and variances of these parameters to be determined.

We developed methods for extracting strain-related data from CCD camera output on ID11 based on similar principles, although some significant differences arose from the two dimensional nature of the collected data. Ideally, an entirely strain-free, fine grained powder sample would produce a diffraction pattern consisting of perfect concentric rings. Macroscopic strain applied to the sample would distort each of these rings into an ellipse, with the orientation of the minor semiaxis coincident with that of the most tensile principal strain, in the plane normal to the beam. Note that a slight orientation mismatch exists between the in-plane direction and the actual diffraction vector. However, for reflections with small indices, at the high energies used in our experiments (around $90 \mathrm{keV}$ ) this error is negligibly small from the engineering viewpoint (about $2^{\circ}$ ). In order to determine the plane strain state, at first sight it is tempting to apply two dimensional fitting directly to the elliptical patterns. In our experience, however, this approach turns out to be unfeasible, for the following reasons. Firstly, no ring measured on a powder sample is ever perfect, due to slight misalignments between the beam and camera axes, irregularities in the pixel positions, etc. Secondly, despite the best efforts to compensate for the known shifts, some systematic distortions remain, which tend to be dominant in determining the ellipse orientation and shape. It is clear, 
however, that these distortions apply to equal extents to both the reference and the measurement rings.

In view of the above considerations, we have adopted an approach whereby each $2 \mathrm{D}$ image is subjected to re-binning into polar coordinates with respect to the image centre. It is found from experience that this centre is best determined from fitting circles to one or several rings in the pattern. It is also clear from simple geometrical analysis that slight errors in its determination do not significantly affect the strain calculations. The radial and angular bin widths can be chosen to minimise the statistical error and maximise the resolution. We have found that radial width of a single pixel and angular width of several degrees $\left(5-10^{\circ}\right)$ give very smooth profiles and reasonable angular variations. For the purposes of error propagation we assumed that each pixel count obeyed Poisson statistics $\left(\sigma_{n}=\sqrt{ } x_{n}\right.$, where $x_{n}$ is pixel intensity), while the values within each bin were distributed according to a Gaussian law, so that the mean and variance for each bin are given by [5]

$$
<x>=\frac{N}{S_{N}}, \quad<\sigma^{2}>=\frac{1}{S_{N}}, \quad \text { where } S_{N}=\sum_{n=1}^{N} 1 / x_{n} .
$$

The radial variation in each angular direction then represents an equivalent line scan, which can be subjected to the standard 1D peak fitting treatment. In our experiments the peak shapes were well represented by superpositions of Gaussian functions. Strain values were obtained from calculated peak centre shifts with respect to the reference peak positions, by using (Eq.1) in which $d$ and $d_{0}$ may be thought of as diffraction ring diameters.

\section{RESULTS}

Figure 1a shows the loading configuration used to produce a contact stress distribution in a SiC fibre-reinforced Ti-6Al-4V composite. An SEM micrograph of the surface containing an indentation produced by the alumina knife in shown in Figure 1b. The strain distributions in the fibre direction in the matrix and the reinforcement are shown in Figures 1c and 1d. For comparison, the contours of equal strain in the direction of the load, as predicted by the Hertzian elastic solution for a homogeneous body, are superimposed on the map for the matrix (Figure 1c), and show good agreement with the measurements. However, the strain map for the reinforcement, shown in Figure $1 \mathrm{~d}$, is significantly different, with the maximum strain measured directly along the line of action of the load, but further below the region of contact.

The results described above may be interpreted qualitatively as follows. Local fibre crushing transfers the load to the matrix, which deforms plastically, redistributing the load over a larger contact area. The eventual strain distribution in the matrix close to the contact region is sufficiently well described by the Hertzian approximation. In the regions further below the contact, however, the compressive load is transferred back to the fibre in a manner characteristic of composite material.

The inset in Figure 4 shows the set of reference axes and the loading device used to produce four point bending in an Al2124 bar specimen reinforced with particulate SiC. In our study of this material we have considered both smooth-surface specimens, and notched bars, containing a pre-

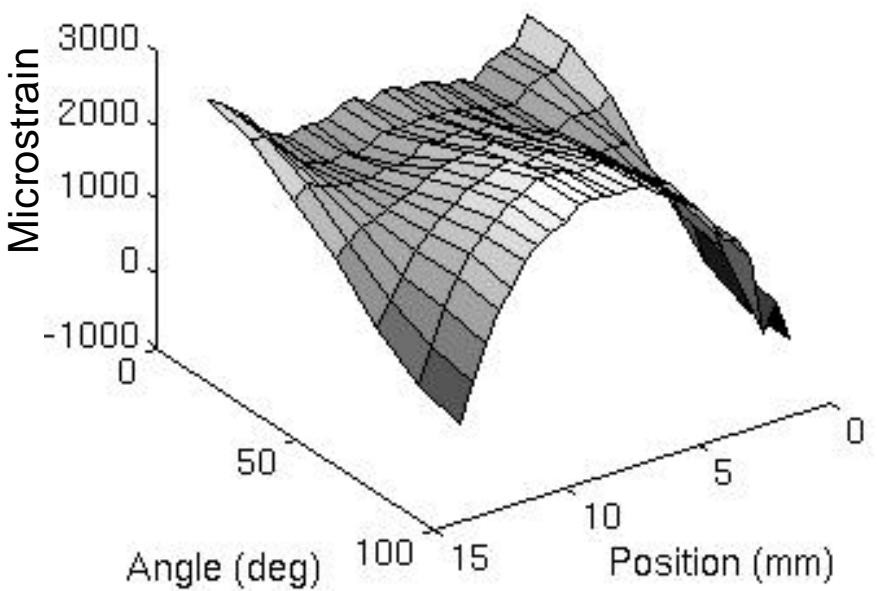

Fig.3. Strain $\varepsilon_{\mathrm{yy}}$ variation in the reference (NA) configuration as a function of position across the specimen ( $x$ coordinate) and the strain measurement orientation. grown fatigue crack.

Figure $2 \mathrm{a}$ shows the evolution of the matrix strain component along the bar length $\left(\varepsilon_{\mathrm{yy}}\right.$, at $90^{\circ}$ to the $\mathrm{x}$-axis) measured at different levels of applied moment: from the quenched and naturally aged (NA) condition, to the strain under high load (HL) inducing significant plastic deformation, to the final unloaded (UL) state. Figure $2 \mathrm{~b}$ shows the matrix strain increment between two measured strain states, (HL-NA), for the $90^{\circ}$ and $0^{\circ}$ strain. The error in the measurement of strain does not exceed the size of the markers used in this figure. The strain resolution is clearly sufficient to reveal the details of the plastic deformation taking place in the matrix, e.g. the asymmetry of yielding in 


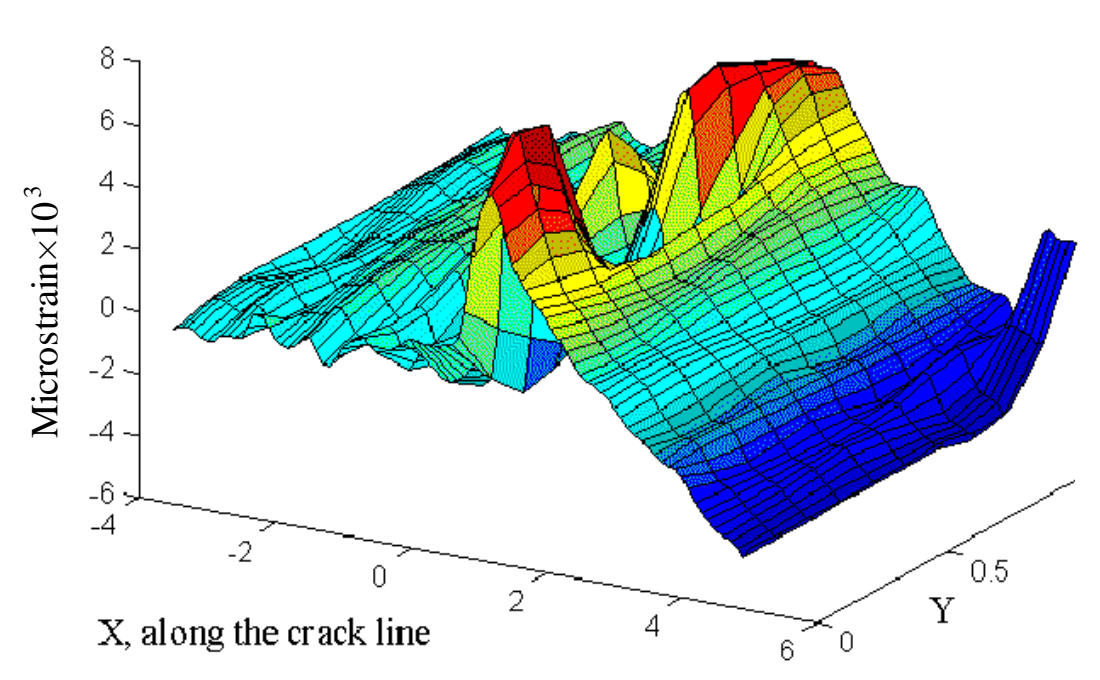

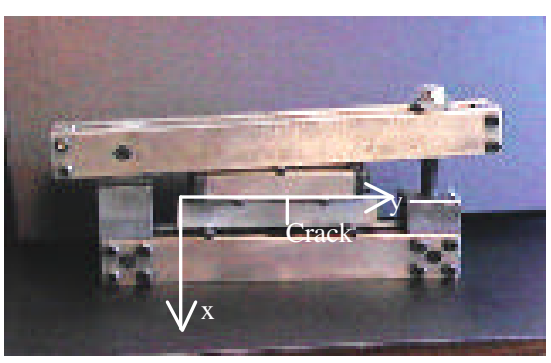

Fig.4. 2D-map of strain $\varepsilon_{\mathrm{yy}}$ in the crack opening direction, showing intensification near the tip and bending strain in the uncracked ligament.

tension and compression, which in this case is mostly caused by the presence of residual stress. It is also interesting to note that no visible deviation from elastic behaviour can be seen in the $0^{\circ}$ strain. The strain increment (HL-UL) is shown in Figure 2c, which indicates that unloading happens elastically.

In order to demonstrate the full extent of the methods capability to extract information on the 2D-strain at a point, we present in Figure 3 a surface plot of the residual strain in the NA condition, as a function of position $y$ across the sample, and the strain measurement orientation.

Finally, as an example of 2D-mapping, Figure 4 shows a surface plot of strain measured in the opening direction in the vicinity of a fatigue crack (which lies at $x<0, y=0.5$ on the plot). The peaks in the vicinity of the tip are characteristic of stress/strain intensification, while the linear variation in the uncracked ligament represents the bending strain profile.

\section{CONCLUSIONS}

The experimental results were presented in this paper in order to outline some possibilities and prospects for the use of synchrotron X-ray diffraction for strain scanning in engineering. Strain measurement procedures were discussed for two particular instrumental configurations, used on the BM16 and ID11 beamlines at the ESRF. The method's versatility was illustrated using the examples of strain 2D-mapping in Ti/SiC fibre reinforced composite, and $2 \mathrm{D}$-strain scanning in $\mathrm{Al} / \mathrm{SiC}$ particulate composite.

\section{ACKNOWLEDGEMENTS}

The authors would like to thank ESRF for the provision of facilities and the University of Newcastle for financial support, and acknowledge the funding from the European Community TMR programme. We would like to thank DERA Sigma and Aerospace Metal Composites (AMC), both of Farnborough, UK, for the provision of fibre reinforced Ti matrix material and particulate reinforced Al composite, respectively. We are grateful to Eric Dooryhee, Andy Fitch, Heinz Graafsma, and Gavin Vaughan, all of ESRF, and to Phil Withers and Alex Owen of the University of Cambridge, for their help in designing and conducting the experiments.

\section{REFERENCES}

[1] A.P. Hammersley, S.O. Svensson, M. Hanfland, A.N. Fitch, and D. Hausermann, High Pressure Research 14, 235 (1996).

[2] G. Vaughan, Private communication (1998).

[3] A.M. Korsunsky, K.E. Wells and P.J. Withers, ESRF Experimental Report HS307 (1997).

[4] A.M. Korsunsky, K.E. Wells and P.J. Withers, Scripta Mat. in press.

[5] P.R. Bevington, D.K. Robinson, Data Reduction and Error Analysis for the Physical Sciences, McGraw-Hill (1992). 